\title{
Cyfip1 haploinsufficiency is associated with white matter changes, myelin thinning, reduction of mature oligodendrocytes and behavioural inflexibility
}

Authors:

Ana I. Silva ${ }^{1,2,3}$, Josephine E. Haddon ${ }^{1,2,4}$, Simon Trent ${ }^{1,2}$, Yasir Ahmed Syed ${ }^{1,5}$, Tzu-Ching E.Lin ${ }^{1,2}$, Yateen Patel ${ }^{1,2}$, Jenny Carter ${ }^{1,2}$, Niels Haan ${ }^{1,2}$, Rob C. Honey ${ }^{4}$, Trevor Humby ${ }^{1,4}$, Yaniv Assaf ${ }^{6}$, David E. J. Linden ${ }^{1,2,3}$, Michael J. Owen ${ }^{1,2}$, Jeremy Hall ${ }^{1,2^{*}}$, Lawrence S. Wilkinson ${ }^{1,2,4 *}$

${ }^{1}$ Neuroscience and Mental Health Research Institute, MRC Centre for Neuropsychiatric Genetics and Genomics, Hadyn Ellis Building, Cathays, Cardiff, CF24 4HQ.

${ }^{2}$ Division of Psychological Medicine and Clinical Neurosciences (DPMCN), School of Medicine, Cardiff University, Cardiff, UK.

${ }^{3}$ Cardiff University Brain Research Imaging Centre School of Psychology, Cardiff University, Maindy Road, Cardiff, CF24 4HQ.

${ }^{4}$ School of Psychology, Cardiff University, Cardiff, Park Place, CF10 3AT

${ }^{5}$ School of Bioscience, The Sir Martin Evans Building, Museum Ave, Cardiff CF10 3AX

${ }^{6}$ Department of Neurobiology, Faculty of Life Sciences, Tel-Aviv University, Ramat Aviv, Tel-Aviv 6997801, Israel;

*Corresponding authors: Lawrence S. Wilkinson \& Jeremy Hall E-mail address: wilkinsonl@cardiff.ac.uk; Hallj10@cardiff.ac.uk Phone number: 029020870357

Address: Neuroscience and Mental Health Research Institute, Hadyn Ellis Building, Cathays, Cardiff, CF24 4HQ. 


\begin{abstract}
The biological basis of the increased risk for psychiatric disorders due to the pathogenic 15q11.2 copy number deletion is unknown. Previous work has shown disturbances in white matter tracts in human carriers of the deletion at this locus. Using a novel rat model, we have recapitulated low dosage of the candidate risk gene CYFIP1 present within the $15 \mathrm{q} 11.2$ interval to reveal alterations in white matter microstructure. Using diffusion tensor imaging, we first showed extensive white matter changes in Cyfipl mutants that were most pronounced in the corpus callosum and external capsule. Transmission electron microscopy showed that these changes were associated with thinning of the myelin sheath in the corpus callosum. Myelin thinning was independent of changes in axon number or diameter but was associated with a reduction in the number of mature oligodendrocytes. Finally, we demonstrated functional effects on cognitive phenotypes sensitive to both disruptions in myelin and callosal circuitry.
\end{abstract}


Low gene dosage of the cytoplasmic FMRP interacting protein 1 (CYFIPl) gene is a candidate risk factor for psychopathology by virtue of its involvement in the pathogenic 15q11.2 BP1-BP2 copy number variant (CNV). Heterozygous deletion of this genomic interval leads to a two- to four fold increase in risk for intellectual disability and psychiatric problems, including schizophrenia, autism, as well as a significant increase in the risk for epilepsy $^{1,2}$. The deletion contains four genes: non-imprinted in Prader-Willi/Angelman syndrome 1 gene (NIPA1), non-imprinted in Prader-Willi/Angelman syndrome 2 gene (NIPA2), CYFIP1, and tubulin gamma complex associated protein 5 gene $(T U B G C P 5)^{3}$. Whilst all these genes are expressed in the brain and may be of potential relevance to psychopathology, CYFIP1 haploinsufficiency is considered to be a likely significant contributor to the 15q11.2 BP1-BP2 psychiatric phenotype due to its known involvement in a number of key brain plasticity-related functions. These include alterations in dendritic spine morphology and branching, mediated by interactions in two distinct complexes: the WAVE regulatory complex to modulate $\mathrm{ARP} 2 / 3$ dependent actin cytoskeleton dynamics, and CYFIP1-eIF4E complex to suppress protein translation at the synapse through interactions with fragile $\mathrm{X}$ mental retardation 1 protein (FMRP), the gene product of $F M R 1^{4}$. Mutations in FMR 1 are causative for fragile $\mathrm{X}$ syndrome, a condition associated with intellectual disability and a range of psychiatric symptoms ${ }^{5}$.

Changes in white matter microstructure have been reported consistently in major psychiatric disorders including schizophrenia, autism and intellectual disability ${ }^{6}$. Moreover, using diffusion tensor imaging (DTI) methods, we found extensive white matter changes in 15q11.2 BP1-BP2 CNV carriers, specifically widespread increases in fractional anisotropy (FA) in deletion carriers (in press) ${ }^{7}$. Some of the biggest changes we observed were in the posterior limb of the internal capsule and corpus callosum. Prominent effects in the corpus 
callosum are consistent with previous findings by others of increased corpus callosum volume in $15 \mathrm{q} 11.2 \mathrm{BP} 1-\mathrm{BP} 2$ deletion subjects ${ }^{8}$. The human data raise three main questions; which of the four genes in the 15q11.2 BP1-BP2 interval are important for the disturbances in white matter microstructure, what are the cellular changes underlying the white matter effects, and what are the functional consequences of the white matter changes in the context of the increased risk for disorder. Given the potential major impact of CYFIP1 in $15 \mathrm{q} 11.2$ BP1-BP2 associated phenotypes, in the present work we addressed these questions by taking advantage of the enhanced experimental tractability of a Cyfipl haploinsufficiency rat line (hereafter designated Cyfip $1^{+/}$) created using CRISPR/Cas9 technology modelling the reduced gene dosage of $C Y F I P 1$ in $15 \mathrm{q} 11.2 \mathrm{BP} 1-\mathrm{BP} 2$ deletion carriers.

Our focus on white matter microstructure was also guided by evidence that CYFIP1 is an actin regulator, and thus likely to affect white matter via the requirement of precise regulation of the actin cytoskeleton for normal cellular development, morphology and migration. Hence, CYFIP1 haploinsufficiency has the potential to disrupt axonal organisation via both effects on axonal guidance ${ }^{9}$ and the myelin component of white matter tracts ${ }^{10,11}$. Myelin is produced by mature oligodendrocytes and several studies have linked actin regulators to oligodendrocyte-myelin dynamics. The Wiskott-Aldrich Syndrome protein family member 1 (WAVE1) and the integrin-linked kinase (ILK) regulate oligodendrocyte differentiation and axon ensheathment ${ }^{12,13}$, while the Arp2/3 complex, a key actin nucleator, is required for initiation of myelination ${ }^{11}$, and Rho GTPases Cdc42 and Rac1 regulate myelin sheath formation ${ }^{14}$.

We therefore hypothesised there would be white matter abnormalities in the Cyfip1 ${ }^{+/-}$rat line possibly linked to underlying changes in axonal architecture including myelin. We also 
anticipated functional effects on brain and behaviour on the basis that axon-myelin perturbations can have marked effects on brain network activity caused by disruptions in the temporal coherence of action potential integration across different brain regions ${ }^{15,16}$. Synchronization of synaptic signals is crucial in learning, and a previous study in shiverer (deletion mutant of myelin basic protein (MBP)) and mld (allelic mutant to shiverer with lowered MBP expression) mice ${ }^{15}$ showed that deficits in myelination had a specific effect on cognitive flexibility in a reversal learning task. In the present work, we looked for evidence of maladaptive brain function in the Cyfip $1^{+/-}$rats using behavioural tasks that assayed cognitive flexibility.

Our findings from in vivo DTI confirmed that Cyfipl haploinsufficiency is associated with white matter microstructure changes, most prominently in the corpus callosum and external capsule. Consistent with our conjecture that Cyfipl haploinsufficiency may influence underlying axon-oligodendrocyte-myelin dynamics, transmission electron microscopy demonstrated that the white matter changes in the corpus callosum were associated with a thinning of the myelin sheath. The effects on myelin were independent of changes in axon number or diameter but were associated with a reduction in the population of mature oligodendrocytes, the specific cell-type that produces myelin. Additionally, we demonstrated changes in cognitive phenotypes required for flexible behavioural responses that were consistent with effects on callosal circuitry and representative of core deficits that are prominent across schizophrenia, autism and fragile $\mathrm{X}$ syndrome ${ }^{17-23}$. 


\section{Results}

\section{Cyfip1 haploinsufficiency disrupts white matter microstructure}

Full details of the creation of the Cyfip1 ${ }^{+/-}$rat model are in the Supplementary Methods.

CRISPR/Cas9 targeting led to a 4bp out of frame heterozygous deletion in exon 7 of the Cyfipl gene at location Chromosome 1: 36974-36977 and a resulting bioinformatics prediction of an early stop codon in exon 8 which was verified functionally using qPCR and Western Blot to measure reductions in mRNA and protein respectively.

To investigate white matter microstructure in the $C y$ fip $^{+/-}$rat brain a cohort of 24 male rats (wild-type (WT) $n=12$, Cyfip $1^{+/-} \mathrm{n}=12$ ) were anaesthetised with isoflurane in oxygen at $4 \%$ and maintained at $1 \%$, and DTI data were collected using a 9.4T MRI scanner, utilising 60 noncollinear gradient directions with a single b-value shell at $1000 \mathrm{~s} / \mathrm{m}^{2}$. Group comparisons were carried out using Tract-Based Spatial Statistics (TBSS) ${ }^{24}$ available in FMRIB Software Library (FSL), with a randomize function allowing voxel-wise nonparametric permutation analysis of the DTI maps projected onto a whole brain white matter skeleton (Supplementary Figure 1). The randomize function was used with the threshold-free cluster enhancement $(\mathrm{TFCE})^{25}$, generating cluster-size statistics based on 1000 random permutations. Figure 1 shows the regions where significant differences in white matter microstructure were found after correction for multiple comparisons. Figure 1a shows the pattern of changes using a highly conservative family-wise error (FWE) correction. This approach showed consistent reductions in FA in the corpus callosum, in the external and internal capsule, and in parts of the fimbria/fornix in Cyfip1 $1^{+/-}$rats, with no differences in axial diffusivity (AD), radial diffusivity (RD), and mean diffusivity (MD). We complemented the highly conservative 
FWE correction method used in human imaging studies with the False Discovery Rate (FDR) correction for multiple comparisons based on the Benjamini-Hochberg procedure ${ }^{26}$ used previously by ourselves ${ }^{27}$ and others ${ }^{28}$ in rodent imaging data. This analysis, shown in Figure $1 \mathrm{~b}$, revealed additional white matter changes including increases in FA in regions of the fornix and fimbria suggesting that Cyfipl haploinsufficiency may have differential effects in different brain regions. Figure $1 \mathrm{~b}$ also shows changes in other DTI metrics, after FDR correction, illustrating mostly decreases in $\mathrm{AD}$ and increases in $\mathrm{RD}$, and MD. These effects were complementary in terms of (a) being localised in the corpus callosum and external and internal capsule and (b) being consistent with the overall predominant effects of Cyfip1 haploinsufficiency in reducing FA. 


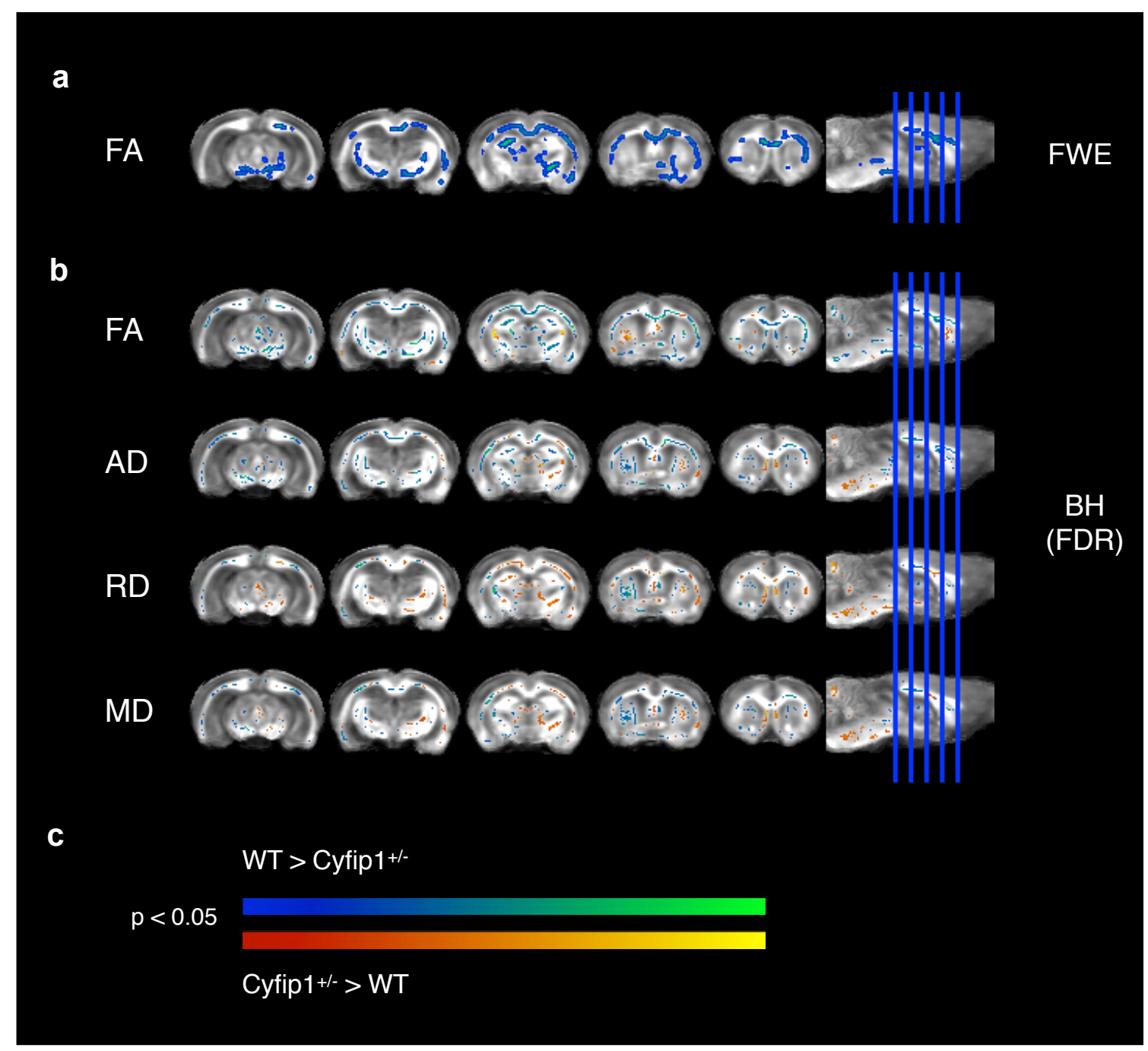

Figure 1 - Cyfip1 haploinsufficiency disrupts white matter microstructure. White matter changes comparing WT $(\mathrm{n}=12)$ and Cyfip1 $1^{+/-}(\mathrm{n}=12)$ rats. Data shows significant group differences using two-tailed unpaired t-tests based on Threshold-Free Cluster Enhancement (TFCE) algorithm after a family-wise error (FWE) rate correction for fractional anisotropy (FA), and $\mathbf{b}$ false discovery rate (FDR) correction based on the Benjamini-Hochberg procedure for FA, axial diffusivity (AD), radial diffusivity (RD) and mean diffusivity (MD). All the parametric maps were generated at a significance level of $p<0.05$. $\mathbf{c}$ Scale bars indicating the direction of the changes in both a) and b), where relative decreases in Cyfip $1^{+/-}$ rats are represented by a gradient of blue (less significant) to green (more significant), and 
relative increases in $C y f i p 1^{+/-}$are represented by a gradient of red (less significant) to yellow (more significant).

We next manually generated binary masks of regions of interest (corpus callosum, internal capsule, external capsule and fimbria/fornix), guided by the results from FWE correction using FSL (Supplementary Figure 2), and assessed mean FA, AD, RD and MD in these white matter tracts. As can be seen in Table 1, analysing the DTI data in this way (which averaged differences between WT and Cyfip $1^{+/-}$rats within a discrete fibre tract, as opposed to the voxel-by-voxel analysis which detected clusters of voxel differences in white matter tracts across the whole brain) showed that the most significant differences were reductions in FA in the corpus callosum $(\mathrm{t}=2.3, \mathrm{df}=20.75, \mathrm{p}<0.05)$ and external capsule $(\mathrm{t}=2.4, \mathrm{df}=22, \mathrm{p}<0.05)$ in the Cyfip $1^{+/-}$rats compared to WT, as assessed with a two-tailed unpaired t-test. These data were consistent with the previous voxel-by-voxel analysis and provided the additional finding that the most extensive white matter changes in the Cyfip $1^{+/-}$rats occurred in these structures.

Table 1 - Quantification of DTI changes in regions where significant differences in FA were seen in TBSS analysis after FWE correction.

\begin{tabular}{|c|c|c|c|c|c|c|c|c|}
\hline \multirow[t]{2}{*}{ ROIs } & \multicolumn{2}{|c|}{ FA } & \multicolumn{2}{|c|}{$\mathrm{AD}\left(10^{-2}\right)$} & \multicolumn{2}{|c|}{$\operatorname{RD}\left(10^{-3}\right)$} & \multicolumn{2}{|c|}{$\operatorname{MD}\left(10^{-3}\right)$} \\
\hline & WT & Cyfip $1^{+/-}$ & WT & Cyfip $1^{+/-}$ & WT & Cyfip $1^{+/-}$ & WT & Cyfip $1^{+/-}$ \\
\hline $\mathrm{CC}$ & $0.49 \pm 0.02$ & $0.46 \pm 0.02 *$ & $0.14 \pm 0.005$ & $0.13 \pm 0.006^{*}$ & $0.69 \pm 0.05$ & $0.71 \pm 0.04$ & $0.92 \pm 0.04$ & $0.91 \pm 0.03$ \\
\hline IC & $0.45 \pm 0.02$ & $0.44 \pm 0.02$ & $0.11 \pm 0.007$ & $0.11 \pm 0.007$ & $0.53 \pm 0.03$ & $0.54 \pm 0.03$ & $0.74 \pm 0.04$ & $0.73 \pm 0.04$ \\
\hline $\mathrm{EC}$ & $0.38 \pm 0.02$ & $0.36 \pm 0.02 *$ & $0.12 \pm 0.004$ & $0.12 \pm 0.003$ & $0.73 \pm 0.03$ & $0.73 \pm 0.02$ & $0.89 \pm 0.03$ & $0.88 \pm 0.02$ \\
\hline $\mathrm{FF}$ & $0.50 \pm 0.03$ & $0.48 \pm 0.02$ & $0.17 \pm 0.008$ & $0.16 \pm 0.004$ & $0.69 \pm 0.06$ & $0.70 \pm 0.04$ & $1.01 \pm 0.06$ & $1.00 \pm 0.03$ \\
\hline
\end{tabular}

Fractional anisotropy (FA), axial diffusivity (AD), radial diffusivity (RD), and mean diffusivity (MD) values from WT and Cyfip $1^{+/-}$rats. Regions of interest (ROIs): corpus callosum (CC), internal capsule (IC), external capsule (EC) and fornix/fimbria (FF). Results obtained using TBSS-based ROI analysis, mean \pm standard deviation, two-tailed unpaired t-test, $*=<0.05$. 


\section{Cyfip1 haploinsufficiency is associated with myelin abnormalities in corpus callosum}

We next investigated the cellular nature of the Cyfipl associated DTI changes. DTI measures can be affected by several factors and previous studies have linked decreases in FA in white matter tracts with less myelin, lower axonal density, axonal damage, or changes in axonal organisation $^{29,30}$. To assess cellular changes, we carried out an ultra-structural analysis, blind to genotype, using transmission electron microscopy and focusing on the corpus callosum, given the DTI data indicating the sensitivity of this structure to Cyfipl haploinsufficiency. The experiment used a new cohort of rats (WT n=5, Cyfip $\left.1^{+-} \mathrm{n}=4\right)$. In order to obtain a representative sample, we sampled 15 regions across the anterior-posterior extent of the corpus callosum encompassing the genu, body and splenium, from sagittal brain sections (representative electron microscopy micrographs in Figure 2a). We measured the number of myelinated and unmyelinated axons, the inner diameter and the outer diameter (including the myelin sheath) of each axon, and then calculated the myelin thickness and the g-ratio (myelin thickness relative to axon diameter, where smaller g-ratios indicate greater myelin thickness) of each myelinated axon (see measures taken in Figure $2 b$ ).

We used linear mixed effects (LME) models to analyse the effect of genotype on axon diameter, g-ratio and myelin thickness, considering variation across animals, whereas a twotailed unpaired t-test was used to compare the number of axons between groups. In this analysis, the myelin thickness was log-transformed since the data followed a log-normal distribution, whereas the other measures followed a normal distribution. No genotype differences were found in the number of unmyelinated $(\mathrm{t}=0.39, \mathrm{df}=5.15, \mathrm{p}=0.71)$ and myelinated $(\mathrm{t}=-0.63, \mathrm{df}=5.72, \mathrm{p}=0.55)$ axons (Figure $2 \mathrm{c})$, or in the diameter of the axons (Figure 2d, LME: $\chi^{2}(1)=0.05, p=0.83$ ), suggesting no differences in axonal density and 
calibre in the corpus callosum of the Cyfip ${ }^{+/-}$rats. The analyses did not show a significant increase in g-ratio when comparing all axons in each group $\left(\operatorname{LME}: \chi^{2}(1)=2.03, p=0.15\right)$, however it revealed a significant reduction in myelin thickness in the Cyfip1 ${ }^{+/}$rats (LME: $\left.\chi^{2}(1)=14.63, p<0.001\right)$, both shown in Figure 2e. The fact that we did not see a significant difference in g-ratio could have resulted from variability in the average of axon diameters within animals in the same group, which is related to g-ratio (Supplementary Figure 3). Furthermore, we needed to consider that the extent of myelination can be related to axon diameter ${ }^{31}$, and whether the effects on the g-ratio were specific to certain sizes of axons. Analysing the g-ratio of axons within specific diameter ranges revealed a significant increased g-ratio in each interval in the $C y f i p 1^{+/-}$rats (Figure 2f), that was more significant in larger axons. These analyses indicate decreased myelin thickness in the corpus callosum of the Cyfip1 $1^{+/-}$rats that is more pronounced in larger axons. 
a

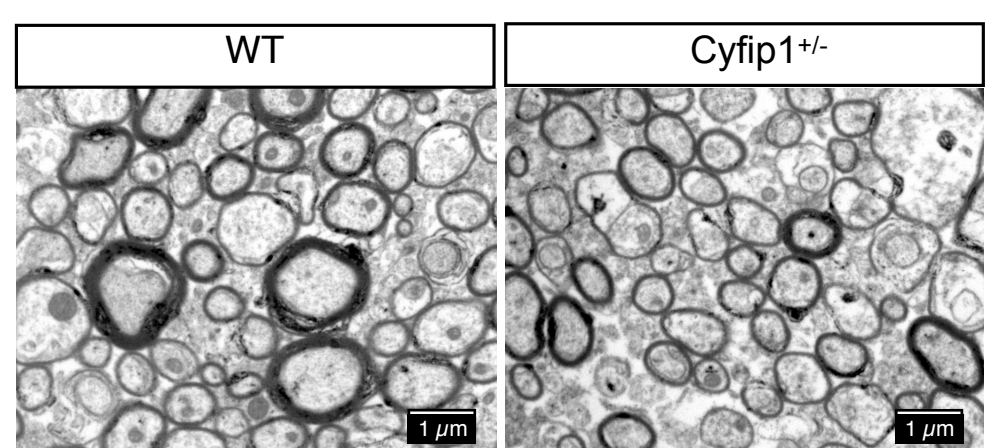

b

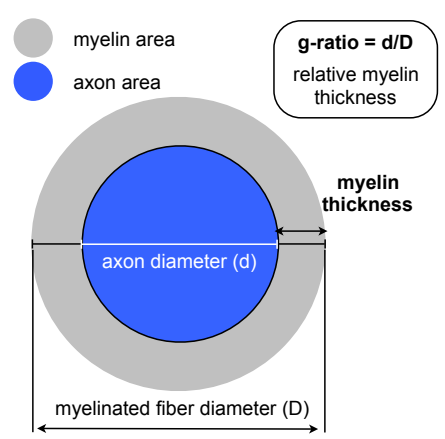

C

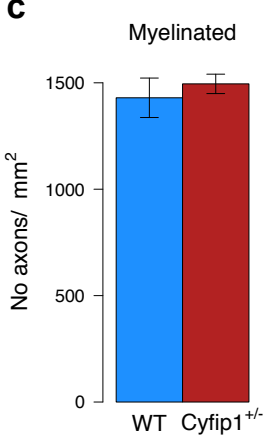

d

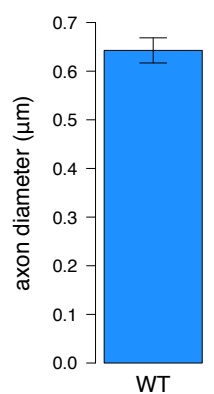

e
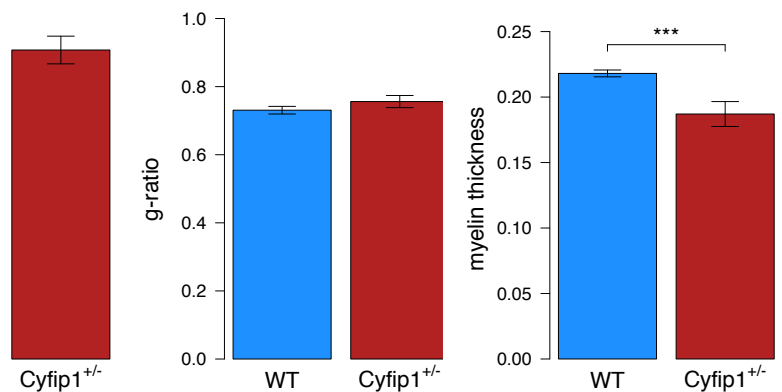

g
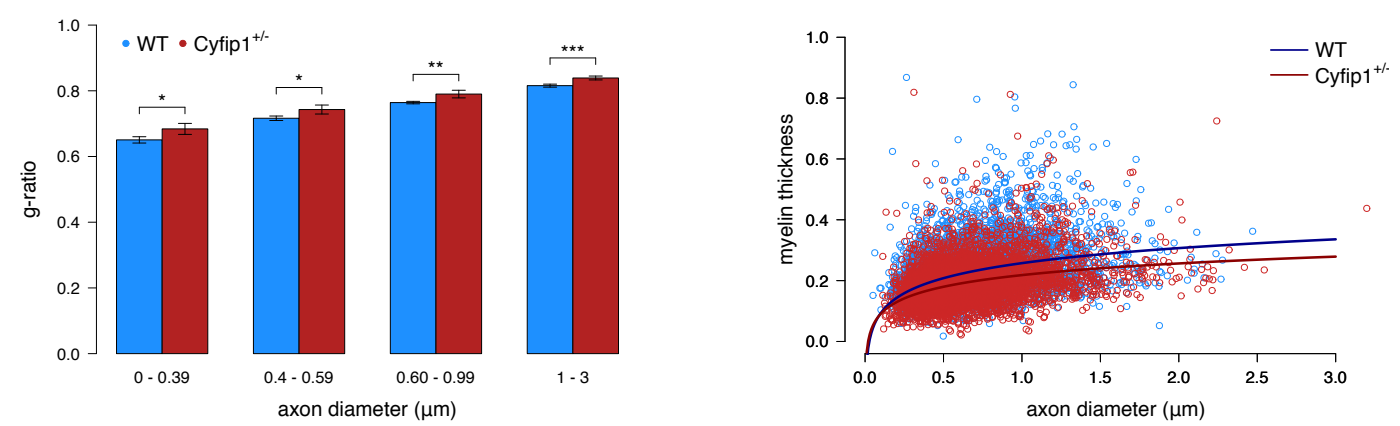

Figure 2 - Decreased myelin thickness in the corpus callosum in the $C y f i p 1^{+/}$rats. a

Representative electron micrographs of axons in the WT ( $\mathrm{n}=5$ animals and $\mathrm{n}=7148$ axons) and Cyfip1 $1^{+/}(\mathrm{n}=4$ animals and $\mathrm{n}=5980$ axons $)$ rats. $\mathbf{b}$ Schematic illustration of the axon and myelin sheath and calculation of the g-ratio and myelin thickness. $\mathbf{c}$ Mean number of myelinated $(\mathrm{t}=-0.63, \mathrm{df}=5.72, \mathrm{p}=0.55, \mathrm{~ns})$ and unmyelinated axons per group $(\mathrm{t}=0.39$, $\mathrm{df}=5.15, \mathrm{p}=0.71, \mathrm{~ns})$, assessed with a two-tailed unpaired t-test. $\mathbf{d}$ Mean axon diameter of myelinated fibres per group (LME: $\left.\chi^{2}(1)=0.05, p=0.83\right)$. e Mean g-ratio per group (LME: $\left.\chi^{2}(1)=2.03, p=0.15\right)$ and mean myelin thickness per group $\left(\operatorname{LME}: \chi^{2}(1)=14.63,{ }^{* * *}\right)$, showing 
significant decreased myelin thickness in Cyfip1 ${ }^{+/-}$rats. f mean g-ratios calculated for small $\left(\mathrm{n}=1510 \mathrm{WT}\right.$ and 1276 Cyfip $^{+/-}$axons; LME: $\left.\chi^{2}(1)=4.23 *\right)$, medium-small $(\mathrm{n}=2283 \mathrm{WT}$ and

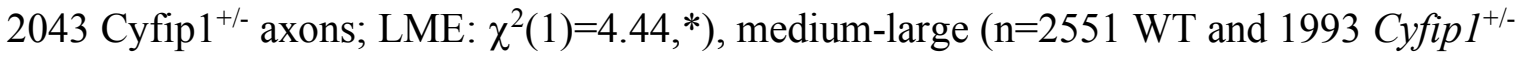
axons; LME: $\left.\chi^{2}(1)=7.14,{ }^{* *}\right)$, and large (n=804 WT and 667 Cyfip $^{+/-}$axons; LME: $\left.\chi^{2}(1)=13.92,{ }^{* * *}\right)$ myelinated axons, showing significant increases in g-ratio in all different axon diameter ranges, and more significant in larger axons. g Scatter plot of myelin thickness values across all axon diameters WT (n=7148 axons) and Cyfipl $1^{+/-}(\mathrm{n}=5980$ axons). Differences between axon diameter, g-ratio and myelin thickness measures were assessed using linear mixed models adjusted for individual variability. Data are mean $\pm \mathrm{SEM} ; *<0.05$, $* *<0.01, * * *<0.001$

\section{Cyfip1 haploinsufficiency leads to reduced numbers of mature oligodendrocytes in corpus callosum/external capsule}

Myelin is produced by mature oligodendrocytes, so we next tested whether Cyfip1 haploinsufficiency influenced the number and/or the maturation of oligodendrocytes using antibodies to the specific molecular markers Olig2 and Cc1. This experiment used rats taken randomly from the same group of rats providing the DTI data shown in Figure 1 (WT $n=7$ WT and Cyfip1 $1^{+/-} \mathrm{n}=7$ ). The analysis focused on the corpus callosum and external capsule and at least four random sections were taken for quantification in each rat from coronal sections. Sections were stained for Olig2 and Cc1 proteins. Cells stained for Olig2 alone represented all the oligodendrocyte lineages from early progenitors to mature cells, whereas cells double-stained for Olig2 and Cc1 proteins revealed specifically the mature oligodendrocyte (myelin-producing) population. In the Cyfip $1^{+/-}$rats, this analysis showed a 
significant reduction in both the number of oligodendrocyte lineage cells (Figure $3 \mathrm{a} ; \mathrm{t}=2.18$, $\mathrm{df}=11.94, \mathrm{p}<0.05)$ and mature oligodendrocytes $(\mathrm{t}=2.48, \mathrm{df}=11.99, \mathrm{p}<0.05)$ in comparison with WT. We also found a marginally significant reduction in the level of myelin basic protein $(\mathrm{MBP})$ (Figure 3b, two-tailed unpaired t-test, $\mathrm{t}=2.16, \mathrm{df}=11.96, \mathrm{p}=0.052)$ in the corpus callosum/external capsule of the $C y f i p 1^{+/-}$rats. These data indicated that $C y f i p 1$ haploinsufficiency resulted in reduced numbers of mature oligodendrocytes. We concluded that these data were consistent with effects of low dosage Cyfipl in reducing myelination that were in turn consistent with the disruptions in white matter microstructure revealed by the DTI analyses.

a

Oligodendrocytes (lineage cells)

Oligodendrocytes (mature cells)

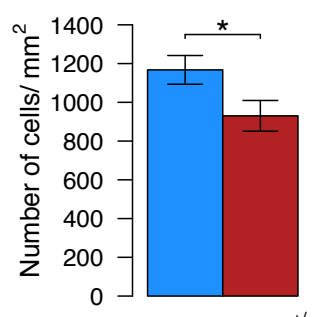

WT Cyfip ${ }^{+1-}$

b

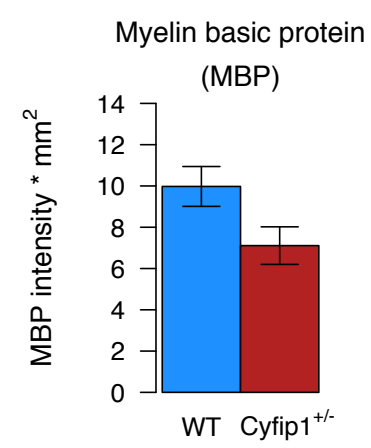

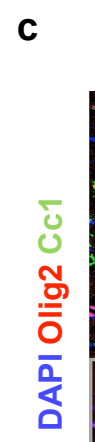

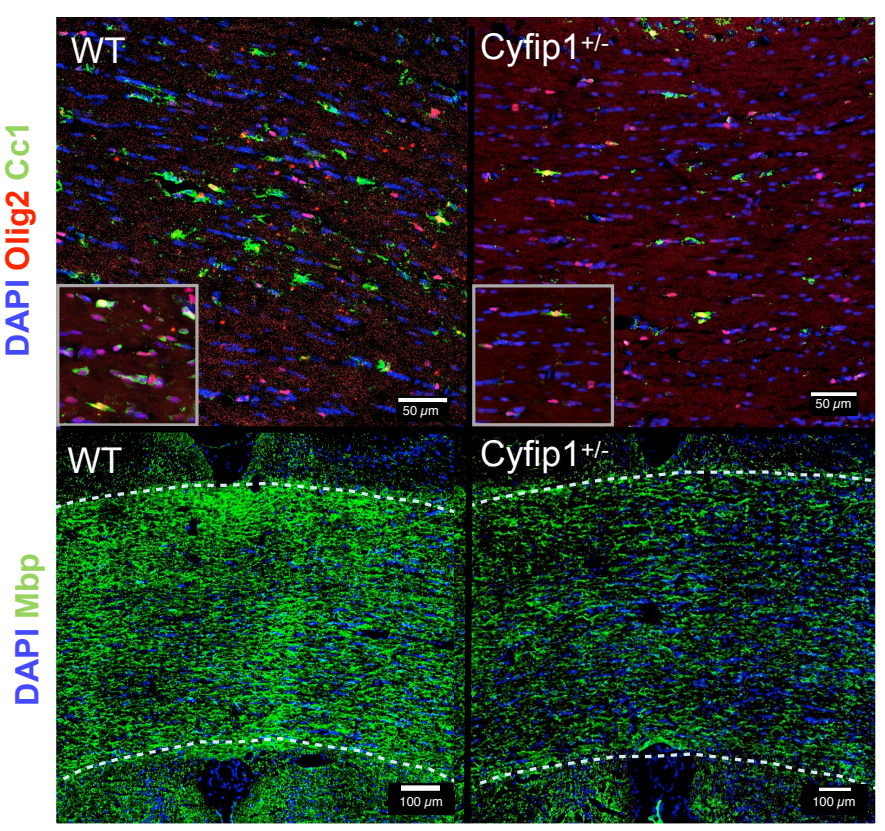

\section{Figure 3 - Decreased number of oligodendrocyte lineage and mature (myelin-}

producing) cells, and decreased level of myelin basic protein (MBP), in the corpus

callosum of the Cyfip1 $^{+/}$rats. a Mean number of oligodendrocyte lineage (n=7 each; $\mathrm{t}=2.18$, $\left.\mathrm{df}=11.94,{ }^{*}\right)$ and mature $\left(\mathrm{n}=7\right.$ each; $\left.\mathrm{t}=2.48, \mathrm{df}=11.99,{ }^{*}\right)$ cells per $\mathrm{mm}^{2} . \mathbf{b}$ Mean MBP 
intensity multiplied by the percentage area $\left(\mathrm{mm}^{2}\right)$ of the staining $(\mathrm{n}=7$ each; $\mathrm{t}=2.16, \mathrm{df}=11.96$, $\mathrm{p}=0.052$ ). c Representative images (at magnification $\mathrm{x} 20$ (top) and $\mathrm{x} 10$ (bottom)) for the following immunomarkers: DAPI, Olig2, Cc1 and Mbp in the corpus callosum of the WT and Cyfip $1^{+/}$rats. Scale bars $=50 \mu \mathrm{m}$ (top) and $100 \mu \mathrm{m}$ (bottom). Data are mean $\pm \mathrm{SEM}$; twotailed unpaired t-test, $*<0.05, * *<0.01, * * *<0.001$.

\section{Cyfip1 haploinsufficient rats show deficits in cognitive flexibility.}

We next assessed whether the Cyfipl related imaging and cellular phenotypes were associated with effects on behaviour. Behavioural changes have been observed in rodent models of reduced myelination including shiverer and $m l d$ mice with modifications in myelin basic protein ${ }^{15}$. Both mutants showed highly specific effects on behavioural flexibility in a reversal learning task, whereby they had difficulty inhibiting a well learned pre-potent response in order to learn a new response, these effects occurring in the absence of any fundamental learning deficits per $s e^{15}$. Furthermore, both human ${ }^{17-19,21-23}$ and animal studies 20,32 have also shown that disruptions to connectivity involving callosal circuitry and circuits involving the internal and external capsules impact on a number of psychological functions, in particular those mediating attention and response control, especially response inhibition.

To assay response inhibition, we utilised a touch screen based appetitive reversal learning task in a separate cohort of experimental rats $\left(\mathrm{WT} n=7, C y f i p 1^{+/-} \mathrm{n}=10\right)$. The reversal learning task first allowed an assessment of basic appetitive learning where rats had to learn that one visual stimulus was associated with reward (the $\mathrm{S}+$ ) and another stimulus was not (S-), the two stimuli counterbalanced across animals. This was followed by reversal of the 
contingencies (see Supplementary Figure 4 for flowchart of task design). Successful reversal is demanding on attention and response inhibition, and can be viewed as a type of "go"-"nogo" discrimination task, similar to those that have been used in clinical studies (i.e. the Stroop task, SART, stop signal) to examine response inhibition ${ }^{33-39}$. All rats successfully completed the early stages of pre-training in the reversal learning task where they had to learn to collect food from the magazine and to touch stimuli presented on the touchscreen to earn rewards, achieving these to criterion in a similar number of sessions (Magazine Training (mean \pm s.e.m): $\mathrm{WT}=2.7 \pm 0.5$, Cyfip $1^{+/-}=3.7 \pm 0.3$, $\operatorname{GENOTYPE}(\mathrm{F}(1,15)=2.79, \mathrm{p}=0.12)$; Touch Training: WT=15.6 $\pm 2.2, C y f i p 1^{+/-}=13.3 \pm 2.1$, GENOTYPE $\left.(F(1,15)=0.53, p=0.48)\right)$. Then the rats moved on to the visual discrimination training where the two stimuli were present. As illustrated in Figure 4a, both groups achieved high levels of performance to a criterion of $80 \%$ correct trials across two successive days and there was no difference in the number of sessions required to reach criterion between the groups (Figure 4b, GENOTYPE $(\mathrm{F}(1,14)=0.03, \mathrm{p}=0.86))$.

Following acquisition of the initial visual discrimination the contingencies were reversed. This manipulation had the expected effect of dramatically reducing correct response as the rats initially persisted in responding to the previously correct but now incorrect (i.e. unrewarded) stimulus leading to 'perseverative' below-chance response. However, and in contrast to the basic learning of the visual discrimination, reversal of the contingencies revealed genotype effects evident in the immediate post-reversal sessions (see Figure 4c). In this part of the task, where inhibition of the previously correct but now incorrect response is the main process controlling behaviour ${ }^{40,41}$, the $C y f i p 1^{+/-}$rats continued to behave according to the previous response strategy showing a significantly greater degree of perseverative, inflexible response, which resulted in them being unable to switch efficiently to the new 
correct response (a 2x2 ANOVA revealed a main effect of $\operatorname{SESSION~}(\mathrm{F}(3,39)=3.67, \mathrm{p}<0.05)$ and a GENOTYPE x SESSION interaction $(F(3,39)=3.76, p<0.05)$, where a main effect of GENOTYPE was marginally significant $(\mathrm{F}(1,13)=4.32, \mathrm{p}=0.06))$. Further analysis of the simple effects of the interaction revealed an effect of SESSION in the WT $(\mathrm{F}(3,39)=7.34$, $\mathrm{p}<0.01)$ but not in the Cyfip $1^{+/-}$group $(\mathrm{F}(3,39)=0.09, \mathrm{p}=0.96)$, and an effect of GENOTYPE on sessions 3 and 4 following reversal (Minimum $F(1,52)=9.42, \mathrm{p}<0.01$ ).

In subsequent sessions with the reversed contingencies the rats were gradually able to inhibit the previous response until they reached the criterion of $50 \%$ correct, where perseverative below chance responses were no longer apparent. However, again, group differences were apparent in those fewer Cyfip1 $1^{+-}$rats that successfully reached this criterion (Figure $4 \mathrm{~d}$, $\left.\chi^{2}=9.61, p<0.05\right)$. Effectively this sub-group of rats were never able to successfully inhibit the previously learned response despite being given ample opportunity to do so that extended to the end of the experiment (rats that completed this phase of reversal did so in an average of 13 sessions, whereas those that failed to learn the reversed contingencies had an average of 22 sessions before the end of the experiment). Figure $4 \mathrm{~d}$ also illustrates the high degree of behavioural specificity shown by the $C y f i p 1^{+/-}$rats in the task; insofar as genotype differences were not evident in the relative proportion of those rats that were able to successfully inhibit the previous response strategy, and went on to learn the new contingency to $80 \%$ criterion. Moreover, Cyfip $1^{+/-}$rats that completed these stages of reversal (reaching $50 \%$ and $80 \%$ correct) did so in a similar number of sessions to WTs (Figure 4e; see also Supplementary Table 1a for the number of rats completing each stage of the reversal task; the total sessions and trials across the whole task are also shown in Table $1 \mathrm{~b}$ ). 
We further assessed the effects of Cyfipl haploinsufficiency on flexible responding in another cohort of rats (WT $n=21$, Cyfip $1^{+/-} \mathrm{n}=15$ ) using an associative mismatch task. This task again assayed visual object recognition and discrimination but in addition allowed a determination of novelty detection and habituation, the latter a form of inhibition distinct from that required in reversal learning tasks. Following habituation to the experimental chambers, the rats received presentations of auditory cues that were predictive of different visual stimuli (i.e. Tone $\rightarrow$ Steady Light, Click $\rightarrow$ Flashing Light; the combinations were randomly counterbalanced across animals). As expected, presentation of the auditory cued visual stimuli resulted in habituation to orienting response toward the light cues over the four training sessions. There were no effects of genotype on behaviour in this part of the task or on habituation to the test apparatus, Figure 5a, where a 2x2 ANOVA on activity during habituation to the chambers revealed a main effects of $\operatorname{BLOCK}(F(5,170)=119.5, p<0.05)$ but no other effect of genotype or interaction (Maximum $\mathrm{F}(5,170)=0.4, \mathrm{p}=0.85$ ). A one-way ANOVA revealed no differences in orienting to the cued visual stimuli at the end of the training phase (Figure 5b, GENOTYPE $(\mathrm{F}(1,34)=2.4, \mathrm{p}=0.62)$ ). In contrast during testing, where the rats were presented with both the original training trials (match) and novel mismatch combinations of the same cues (e.g. Tone $\rightarrow$ Flashing Light, Click $\rightarrow$ Steady Light), the Cyfip $1^{+/-}$rats failed to show the normal preference of orienting to the novel mismatch cues relative to match cues ${ }^{42}$, as demonstrated by the WT rats, instead showing no preference for responding to either the habituated or the novel cues (Figure 5c, GENOTYPE $(\mathrm{F}(1,34)=5.92, \mathrm{p}<0.05)$ as indicated by the discrimination ratios (total orienting to matched / total orienting to both matched + mismatched). This pattern of behaviour was further evidence for an inhibitory deficit associated with Cyfipl haploinsufficiency where prior learning, in this case the matching cues, dominates current responding leading to maladaptive and inflexible behaviour. 


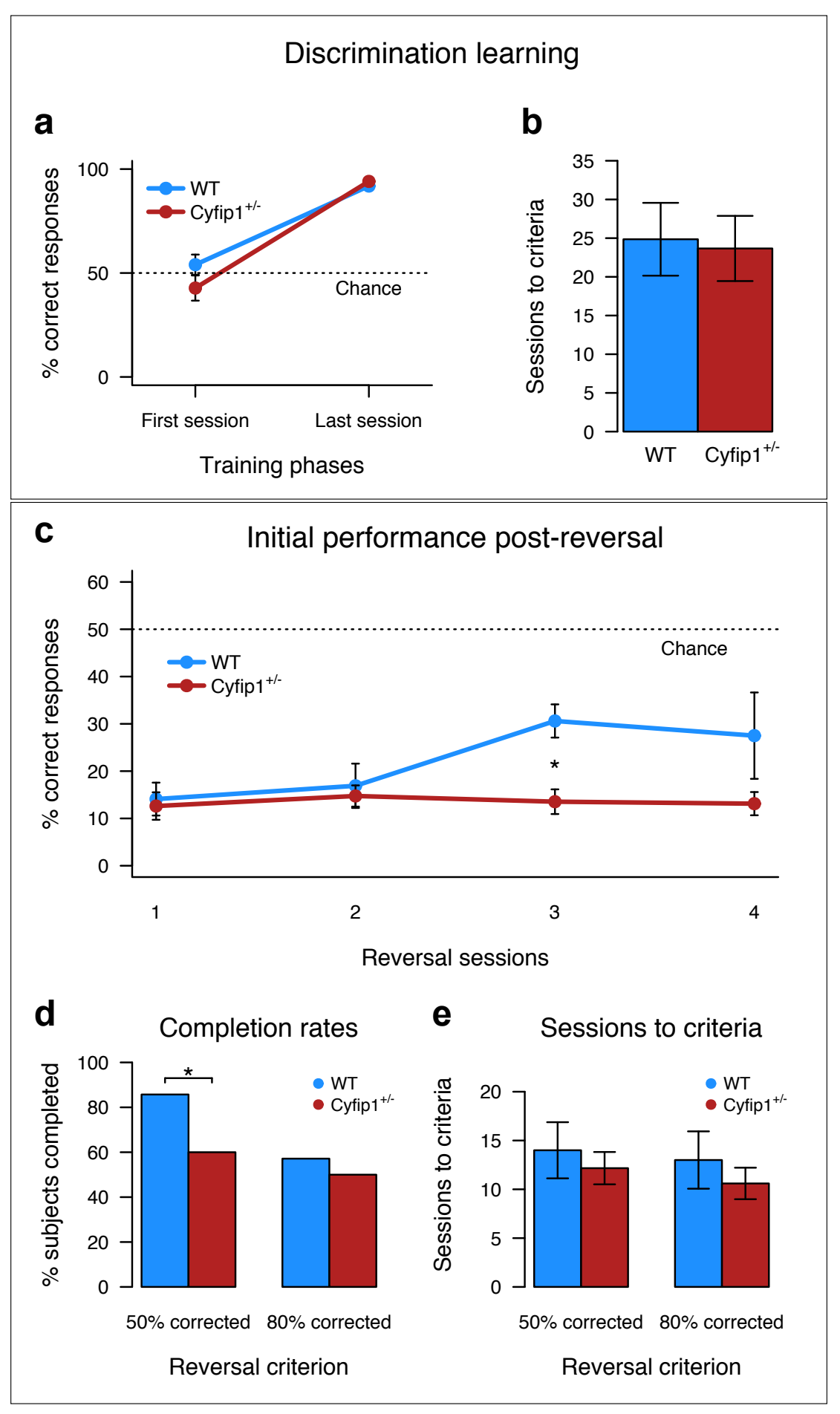

Figure 4 - Cyfip1 haploinsufficient rats show deficits in behavioural flexibility in a

reversal learning paradigm. WT $(n=7)$ and $C y f i p 1^{+/-}(n=10)$ rats successfully acquire the visual discrimination in the touchscreen boxes, reaching the same level of performance $(\%$ 
correct) a during the last session of training, and $\mathbf{b}$ reaching criteria in a similar number of sessions $(\mathrm{F}(1,14)=0.03, \mathrm{p}=0.86)$. $\mathbf{c}$ Cyfip1 ${ }^{+/-}$rats show more persistent responses to the original stimulus response contingencies during the first few sessions of reversal, (GENOTYPE X SESSION interaction, $\left.\mathrm{F}(3,39)=3.76,{ }^{*}\right)$. d A larger proportion of Cyfip1 ${ }^{+/-}$ rats failed to reach the $50 \%$ correct criterion during reversal than WT rats, $\left(\chi^{2}=9.61,{ }^{*}\right)$. e However, those that do reach criteria do so in a similar number of sessions as WT rats. Data are mean \pm SEM. $*<0.05, * *<0.01, * * *<0.001$. See also Supplemental Table 1 for additional data.

a

Habituation

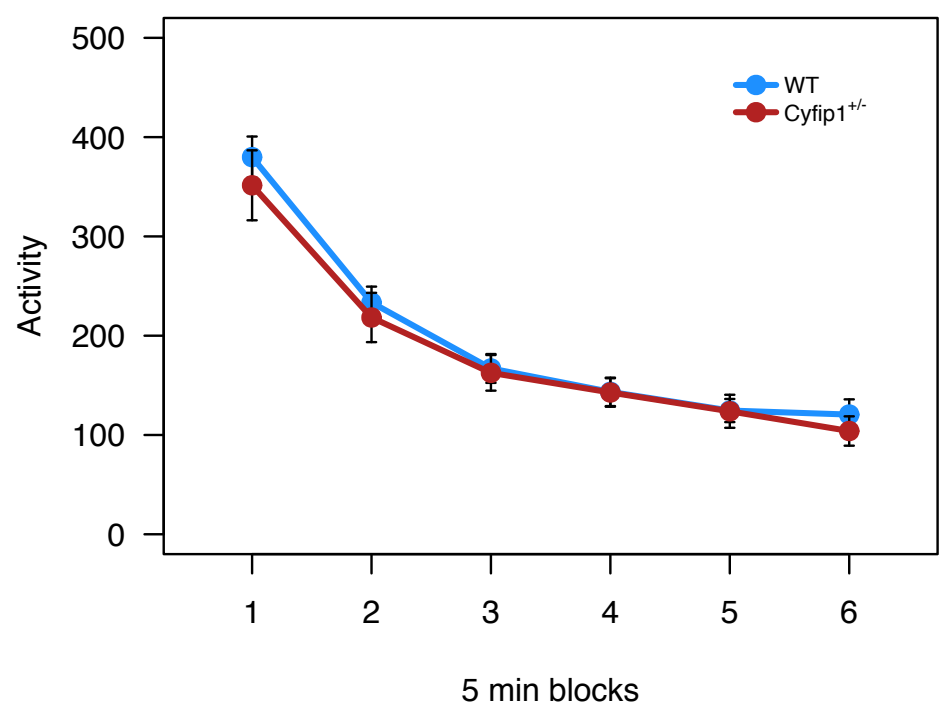

b

Orienting response

C Mismatch test
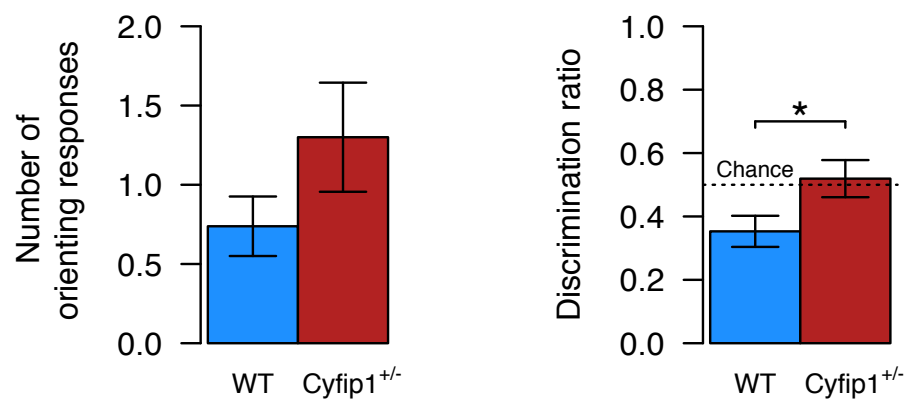


\section{Figure 5 - Cyfip1 haploinsufficient rats show deficits in flexible responses in orienting}

behaviour on a mismatch task. a There are no genotype effects on habituation to the experimental apparatus, both WT $(\mathrm{n}=21)$ and Cyfip1 $^{+/-}(\mathrm{n}=15)$ show reduced activity to the experimental chambers over the course of each 30 minute long session ( 5 min blocks). b Both WT and Cyfipl ${ }^{+/-}$rats showed reduced orienting to the auditory-visual sequences presented during training $(\mathrm{F}(1,34)=2.4, \mathrm{p}=0.62)$, and $\mathbf{c} \mathrm{WT}$ rats preferentially responded to the novel mismatched visual stimuli over the familiar matched stimuli (lower discrimination ratio than chance (0.5)). In contrast, $C y f i p 1^{+/-}$rats showed no preference, responding equally to both matched and mismatched cues $\left(\mathrm{F}(1,34)=5.92,{ }^{*}\right), *<0.05, * *<0.01, * * *<0.001$.

\section{Discussion}

We used a CRISPR/Cas9 engineered rat line to model the contribution of Cyfip1 haploinsufficiency to white matter changes observed in carriers of the pathogenic $15 q 11.2$ BP1-BP2 deletion. The Cyfip1 $1^{+/-}$rat model allowed us to carry out a DTI analysis with high resolution using identical preprocessing to our 15q11.2 BP1-BP2 human imaging study, and employ rigorous statistics including exploratory voxel-wise assessments permitting comparisons of genotype effects across brain regions. The $C y f i p 1^{+/-}$rat line provided enhanced experimental tractability in terms of direct access to brain tissue, with interpretation of DTI changes at the cellular level, and also allowed relevant behavioural analyses under controlled conditions.

A main finding of the DTI experiments were significant decreases in FA that were most pronounced in the corpus callosum and external capsule. These data were obtained using a highly conservative correction procedure. More widespread changes in white matter were 
apparent when using a less conservative correction method including increased FA in some areas of the fornix/fimbria. An advantage of using the Cyfip1 $1^{+/-}$rat model was that we could make a direct assessment of possible cellular changes underlying the DTI effects. The precise relationship between DTI measures and cellular changes is subject to ongoing debate ${ }^{30}$ and whilst DTI can identify white matter changes it cannot definitively distinguish between disruptions to axons and/or myelin ${ }^{43}$. Consequently, at the outset the DTI effects we obtained in the rat model could have been related to changes in axon microstructure or myelin, or both.

A comprehensive quantitative transmission electron microscopy analysis indicated a thinning of the myelin sheath in the corpus callosum of the Cyfip $1^{+/-}$rats in the absence of any changes in axonal number or diameter. Here our observations of a reduction of oligodendrocytes in the corpus callosum and external capsule of the Cyfip $1^{+/-}$rats may be of significance. Myelin is produced only by mature oligodendrocytes and as indexed by the specific marker $\mathrm{CC} 1$, we found significant reductions in these cells. However, as we also found a similar reduction in Olig2, which is a general marker for the oligodendrocyte lineage from early progenitors through to mature cells, it is unlikely that any effects on myelin thickness were the result of a specific deficit in the final stage of commitment to the mature, myelin producing oligodendrocytes. Reductions in oligodendrocyte number have been shown to be associated with myelin thinning ${ }^{44,45}$, and in the case of Cyfipl haploinsufficiency the data allow several potential mechanisms for this, including oligodendrocyte production, differentiation and migration; additionally, effects on neurons can have an impact on axon-oligodendrocyte interactions and affect myelination ${ }^{46}$. Investigation of these possibilities would require the implementation of models that combine low dosage of Cyfipl with gene constructs which allow the labelling of oligodendrocytes at specific stages of the oligodendrocyte life-cycle, such as inducible Sox10 systems ${ }^{47}$. 
The effects of Cyfip1 haploinsufficiency on oligodendrocytes and myelination may be related to core deficits in actin physiology, and previous data from $C y f i p 1^{+/-}$mouse models have shown abnormal f-actin dynamics ${ }^{48}$. As noted previously, a main function of CYFIP1 protein is to inhibit ARP2/3 dependent actin cytoskeleton remodelling, via inhibition of WAVE1 protein and other members of the WAVE regulatory complex. It is known that multiple aspects of oligodendrocyte function, including cell proliferation, differentiation and migration are critically reliant on effective cytoskeleton remodelling ${ }^{10}$. Furthermore, alterations of the cytoskeleton are required to produce myelin, being the formation of lamellipodia and lamellipodial 'ruffles', that make initial contact between the oligodendrocyte and axon, required for myelination to occur ${ }^{49}$. It has also been shown in mouse models that manipulating WAVE1 protein directly impacts on the ability to form myelin, and interestingly these effects were localised in the corpus callosum and not present in other areas of the central nervous system ${ }^{12}$. The extent to which the current effects of Cyfipl haploinsufficiency on oligodendrocyte function are consistent with the effects of WAVE1 manipulation remains to be determined. Effects related to the close interaction between CYFIP1 and FMRP cannot be discounted, especially in view of a degree of overlap between white matter changes in the Cyfip $1^{+/}$rat model and a mouse Fmrl knockout, specifically reduced FA in the corpus callosum ${ }^{50}$. The mouse Fmrl knockout also revealed evidence of global disruptions in functional connectivity. Assuming a role for Cyfip1-Fmrp interactions, the relevant molecular and cellular mechanisms would presumably involve perturbations to synaptic protein homeostasis, however the precise relationship between such effects on synaptic protein dynamics and the phenotypes we observed in our rat model remain to be identified. 
We assessed the extent to which the imaging and cellular data were associated with functional behavioural effects. Behavioural flexibility has been shown to be sensitive to abnormalities in myelination in mouse models of myelin deficits ${ }^{15}$. Furthermore, in humans, corpus callosum morphology was shown to correlate with response inhibition in a study using a cohort of twin pairs, where one was diagnosed with bipolar disorder and the other was clinically healthy ${ }^{17}$. We therefore focused the behavioural analyses on psychological processes supporting behavioural flexibility. In a first experiment assaying reversal learning, we showed that the Cyfip $1^{+/-}$rats perseverated, meaning they continued to respond to stimuli that had resulted in a correct response previously but, following reversal, were now associated with an incorrect response. The effects in the Cyfip1 ${ }^{+/-}$rats were highly specific to the reversal element of the task with no effects on initial learning, and indicated the absence of any generalised learning deficits. Instead, the pattern of data suggested a deficit in the ability to flexibly manipulate previous learning to adapt to changing contingencies.

To extend these behavioural data, and to exclude the possibility of task-specific effects, we conducted an associative mismatch task in a separate cohort of rats that examined a different type of behavioural flexibility, but also requiring inhibition. The associative mismatch task has been less comprehensively specified in terms of underlying neurobiology, with some evidence of involvement of hippocampal circuitry ${ }^{42}$, but it requires attentional, memory and inhibitory processes that have been shown to be mediated by callosal circuitry ${ }^{51-53}$. We again observed highly specific effects on inhibition of previous learning and no effects on learning per se. Together, the data from the two tasks provided converging evidence for an inhibitory deficit associated with Cyfipl haploinsufficiency where prior learning dominates current responses leading to maladaptive inflexible behaviour. An inability to alter behaviour in response to changes in the environment has been strongly associated with ventral prefrontal 
cortex damage in humans ${ }^{54}$ and rats ${ }^{55,56}$. The corpus callosum (along with the internal capsule) carries white matter bundles containing axons projecting from the prefrontal cortex and striatal regions ${ }^{19}$, and it was shown that pruning and myelination of the corpus callosum coincides with cortical maturation in the prefrontal cortex, mutually influencing each other's development ${ }^{57,58}$.

Increases in FA with little evidence of reductions were a prominent feature of our findings in human 15q11.2 BP1-BP2 deletion carriers (in press) ${ }^{7}$. Hence, while the rat model and human phenotype converged on white matter changes they differed in the direction of the change. Differences between the human and rat findings could have resulted from several factors. First, the 15q11.2 BP1-BP2 deletion involves three other genes in addition to CYFIP1, especially NIPA1 which is expressed in the brain and was found to inhibit the bone morphogenic protein (BMP), crucial for typical axonal growth and guidance ${ }^{59,60}$. Therefore, $a$ priori, haploinsufficiency of NIPA, and possibly the other genes in the interval ${ }^{61,62}$, may impact on the 15q11.2 BP1-BP2 deletion DTI phenotype. The possibility that there are species differences in the expression patterns of CYFIP1/Cyfip1 and also any compensatory responses to haploinsufficiency should also be borne in mind. Furthermore, the humans and rats are likely to have been subject to differential compensatory mechanisms arising from very different environmental challenges across their lifespan ${ }^{6,63,64}$. Moreover, as changes in myelin thickness impact relatively modestly on DTI measures ${ }^{30}$ it may be that whilst myelin changes may be present in both human and the rat model, in terms of the human DTI data any effect on myelin may have been masked by other molecular and cellular consequences of the copy number deletion. To date, there have been no published studies of myelin (as opposed to overall white matter) changes in 15q11.2 BP1-BP2 deletion, though the current data predicts their existence and this is something that could be tested using ultrastructural magnetic 
resonance imaging (MRI) methods providing the necessary resolution to visualise and quantify myelinated axons directly in the living human brain ${ }^{65}$. Nonetheless, whilst an exact between-species comparison would require an assessment of CYFIPl-specific heterozygous humans, we have demonstrated a clear link between Cyfipl haploinsufficiency and white matter microstructure.

In conclusion, we have employed a novel rat model of Cyfipl haploinsufficiency to probe the neurobiological and behavioural mechanisms underlying the significantly enhanced risk for psychopathology linked to the 15q11.2 BP1-BP2 deletion. We found disturbances to white matter as seen in human carriers, and showed effects on myelin thickness and a reduction in mature oligodendrocyte number, together with evidence of highly specific behavioural deficits due to maladaptive inhibition. The imaging phenotype on both rats and humans further suggest that it is unlikely that effects mediated by CYFIPI are solely responsible, and additional work is required to determine the contribution made by the other three genes, NIPA1, NIPA2, and TUBGCP5 affected in the 15q11.2 BP1-BP2 deletion. However these findings on the Cyfipl rat model give an insight into the contribution made by low dosage of CYFIP1 to the 15q11.2 BP1-BP2 deletion phenotype. 


\section{Acknowledgements}

This work was also supported by a Wellcome Trust Strategic Award 'DEFINE' grant no. 100202/Z/12/Z and core support from the Neuroscience and Mental Health Research Institute, Cardiff University, UK. We acknowledge technical support for the DTI studies from Andrew Stewart, School of Biosciences, Cardiff University and excellent animal husbandry and care by the Joint Biological Services Unit personal at Cathays Animal Facility, Cardiff University. We also acknowledge technical support for the transmission electron microscopy acquisition from Dr. Christopher Von Ruhland, Central Biotechnology Services, Cardiff University.

\section{Author's contribution}

AIS, JH, DEJL and LSW planned, designed and instigated the study. ST conducted the molecular specification of the novel heterozygous Cyfipl rat line. YP, YA and JC conducted the DTI imaging acquisition, and AIS, YA, DEJL, JH and LSW analysed the DTI data. AIS, $\mathrm{NH}$ and YS obtained and analysed the transmission electron microscopy data and immunofluorescence data. JEH, AIS, JH, TEL, RCH, TH and LSW carried out and analysed the behavioural studies. AIS, JEH, JH, MJO, DEJL and LSW wrote and reviewed the manuscript.

\section{Competing financial interests}

The authors declare no competing financial interests.

\section{Material and correspondence}

All data from this study are available from the corresponding author upon reasonable request. 


\section{References}

1. Cox, D. M. \& Butler, M. G. The 15q11.2 BP1-BP2 Microdeletion Syndrome: A Review. Int. J. Mol. Sci. 16, 4068-4082 (2015).

2. Butler, M. G. Clinical and genetic aspects of the 15q11.2 BP1-BP2 microdeletion disorder. J. Intellect. Disabil. Res. 61, 568-579 (2017).

3. Chai, J.-H. et al. Identification of Four Highly Conserved Genes between Breakpoint Hotspots BP1 and BP2 of the Prader-Willi/Angelman Syndromes Deletion Region That Have Undergone Evolutionary Transposition Mediated by Flanking Duplicons. Am. J. Hum. Genet. 73, 898-925 (2003).

4. De Rubeis, S. et al. CYFIP1 Coordinates mRNA Translation and Cytoskeleton Remodeling to Ensure Proper Dendritic Spine Formation. Neuron 79, 1169-1182 (2013).

5. Lozano, R., Rosero, C. A. \& Hagerman, R. J. Fragile X spectrum disorders. Intractable Rare Dis. Res. 3, 134-146 (2014).

6. Fields, R. D. White matter in learning, cognition and psychiatric disorders. Trends Neurosci. 31, 361-370 (2008).

7. Silva, A. I. et al. Reciprocal white matter changes associated with copy number variation at 15q11.2 BP1-BP2 - A diffusion tensor imaging study. Biol. Psychiatry 0, (2018).

8. Ulfarsson, M. O. et al. 15q11.2 CNV affects cognitive, structural and functional correlates of dyslexia and dyscalculia. Transl. Psychiatry 7, e1 109 (2017).

9. Dent, E. W. \& Gertler, F. B. Cytoskeletal Dynamics and Transport in Growth Cone Motility and Axon Guidance. Neuron 40, 209-227 (2003).

10. Bauer, N. G., Richter-Landsberg, C. \& Ffrench-Constant, C. Role of the oligodendroglial cytoskeleton in differentiation and myelination. Glia 57, 1691-1705 (2009). 
11. Zuchero, J. B. et al. CNS myelin wrapping is driven by actin disassembly. Dev. Cell 34, $152-167$ (2015).

12. Kim, H.-J. et al. WAVE1 Is Required for Oligodendrocyte Morphogenesis and Normal CNS Myelination. J. Neurosci. 26, 5849-5859 (2006).

13. O’Meara, R. W. et al. Integrin-Linked Kinase Regulates Process Extension in Oligodendrocytes via Control of Actin Cytoskeletal Dynamics. J. Neurosci. 33, 97819793 (2013).

14. Thurnherr, T. et al. Cdc42 and Rac1 Signaling Are Both Required for and Act Synergistically in the Correct Formation of Myelin Sheaths in the CNS. J. Neurosci. 26, 10110-10119 (2006).

15. Inagawa, K., Watanabe, S., Tsukada, Y.\& Mikoshiba, K. The role of myelination in learning performance observed in two strains of myelin-deficient mutant mice (shiverer and mld). Behav. Neural Biol. 50, 184-192 (1988).

16. Fields, R. D. A new mechanism of nervous system plasticity: activity-dependent myelination. Nat. Rev. Neurosci. 16, 756-767 (2015).

17. Bearden, C. E. et al. Mapping Corpus Callosum Morphology in Twin Pairs Discordant for Bipolar Disorder. Cereb. Cortex 21, 2415-2424 (2011).

18. Lochner, C. et al. Evidence for fractional anisotropy and mean diffusivity white matter abnormalities in the internal capsule and cingulum in patients with obsessive-compulsive disorder. J. Psychiatry Neurosci. JPN 37, 193-199 (2012).

19. Haber, S. N. \& Behrens, T. E. J. The Neural Network Underlying Incentive-Based Learning: Implications for Interpreting Circuit Disruptions in Psychiatric Disorders. Neuron 83, 1019-1039 (2014). 
20. Magnuson, M. E., Thompson, G. J., Pan, W.-J. \& Keilholz, S. D. Effects of Severing the Corpus Callosum on Electrical and BOLD Functional Connectivity and Spontaneous Dynamic Activity in the Rat Brain. Brain Connect. 4, 15-29 (2013).

21. Alm, K. H., Rolheiser, T., Mohamed, F. B. \& Olson, I. R. Fronto-temporal white matter connectivity predicts reversal learning errors. Front. Hum. Neurosci. 9, (2015).

22. Balevich, E. C. et al. Corpus callosum size and diffusion tensor anisotropy in adolescents and adults with schizophrenia. Psychiatry Res. Neuroimaging 231, 244-251 (2015).

23. Onnink, A. M. H. et al. Deviant white matter structure in adults with AttentionDeficit/Hyperactivity Disorder points to aberrant myelination and affects neuropsychological performance. Prog. Neuropsychopharmacol. Biol. Psychiatry 63, 14 $22(2015)$.

24. Smith, S. M. et al. Tract-based spatial statistics: Voxelwise analysis of multi-subject diffusion data. NeuroImage 31, 1487-1505 (2006).

25. Smith, S. M. \& Nichols, T. E. Threshold-free cluster enhancement: Addressing problems of smoothing, threshold dependence and localisation in cluster inference. NeuroImage 44, 83-98 (2009).

26. Benjamini, Y. \& Hochberg, Y. Controlling the False Discovery Rate: A Practical and Powerful Approach to Multiple Testing. J. R. Stat. Soc. Ser. B Methodol. 57, 289-300 (1995).

27. Sagi, Y. et al. Learning in the Fast Lane: New Insights into Neuroplasticity. Neuron 73, 1195-1203 (2012).

28. Sierra, A. et al. Diffusion tensor MRI with tract-based spatial statistics and histology reveals undiscovered lesioned areas in kainate model of epilepsy in rat. Brain Struct. Funct. 216, 123-135 (2011). 
29. Le Bihan, D. Molecular diffusion, tissue microdynamics and microstructure. $N M R$ Biomed. 8, 375-386 (1995).

30. Beaulieu, C. The basis of anisotropic water diffusion in the nervous system - a technical review. NMR Biomed. 15, 435-455 (2002).

31. Klingseisen, A. \& Lyons, D. A. Axonal Regulation of Central Nervous System Myelination: Structure and Function. The Neuroscientist 24, 7-21 (2018).

32. Coizet, V. et al. Organization of the Anterior Limb of the Internal Capsule in the Rat. $J$. Neurosci. 37, 2539-2554 (2017).

33. Waltz, J. A. \& Gold, J. M. Probabilistic reversal learning impairments in schizophrenia: Further evidence of orbitofrontal dysfunction. Schizophr. Res. 93, 296-303 (2007).

34. Kaland, N., Smith, L. \& Mortensen, E. L. Brief Report: Cognitive Flexibility and Focused Attention in Children and Adolescents with Asperger Syndrome or HighFunctioning Autism as Measured on the Computerized Version of the Wisconsin Card Sorting Test. J. Autism Dev. Disord. 38, 1161-1165 (2008).

35. Dickson, P. E. et al. Effects of stimulus salience on touchscreen serial reversal learning in a mouse model of fragile X syndrome. Behav. Brain Res. 252, 126-135 (2013).

36. Hamilton, D. A. \& Brigman, J. L. Behavioral flexibility in rats and mice: Contributions of distinct frontocortical regions. Genes Brain Behav. 14, 4-21 (2015).

37. Uddin, L. Q. et al. Brain State Differentiation and Behavioral Inflexibility in Autism. Cereb. Cortex N. Y. NY 25, 4740-4747 (2015).

38. Lancaster, T. M. et al. Associations between polygenic risk for schizophrenia and brain function during probabilistic learning in healthy individuals. Hum. Brain Mapp. 37, 491500 (2016). 
39. Reddy, L. F., Waltz, J. A., Green, M. F., Wynn, J. K. \& Horan, W. P. Probabilistic Reversal Learning in Schizophrenia: Stability of Deficits and Potential Causal Mechanisms. Schizophr. Bull. 42, 942-951 (2016).

40. Gabriel, M. \& Orona, E. Parallel and serial processes of the prefrontal and cingulate cortical systems during behavioral learning. Brain Res. Bull. 8, 781-785 (1982).

41. Bussey, T. J., Muir, J. L., Everitt, B. J. \& Robbins, T. W. Dissociable effects of anterior and posterior cingulate cortex lesions on the acquisition of a conditional visual discrimination: Facilitation of early learning vs. impairment of late learning. Behav. Brain Res. 82, 45-56 (1996).

42. Honey, R. C., Watt, A. \& Good, M. Hippocampal Lesions Disrupt an Associative Mismatch Process. J. Neurosci. 18, 2226-2230 (1998).

43. Aung, W. Y., Mar, S. \& Benzinger, T. L. Diffusion tensor MRI as a biomarker in axonal and myelin damage. Imaging Med. 5, 427-440 (2013).

44. Boyd, A., Zhang, H. \& Williams, A. Insufficient OPC migration into demyelinated lesions is a cause of poor remyelination in MS and mouse models. Acta Neuropathol. (Berl.) 125, 841-859 (2013).

45. Syed, Y. A. et al. Inhibition of phosphodiesterase-4 promotes oligodendrocyte precursor cell differentiation and enhances CNS remyelination. EMBO Mol. Med. 5, 1918-1934 (2013).

46. Barres, B. A. \& Raff, M. C. Axonal Control of Oligodendrocyte Development. J. Cell Biol. 147, 1123-1128 (1999).

47. Shibata, S. et al. Sox10- Venus mice: a new tool for real-time labeling of neural crest lineage cells and oligodendrocytes. Mol. Brain 3, 31 (2010). 
48. Pathania, M. et al. The autism and schizophrenia associated gene CYFIP1 is critical for the maintenance of dendritic complexity and the stabilization of mature spines. Transl. Psychiatry 4, e374 (2014).

49. Asou, H., Hamada, K. \& Sakota, T. Visualization of a single myelination process of an oligodendrocyte in culture by video microscopy. Cell Struct. Funct. 20, 59-70 (1995).

50. Haberl, M. G. et al. Structural-functional connectivity deficits of neocortical circuits in the Fmr1-/y mouse model of autism. Sci. Adv. 1, e1500775 (2015).

51. Banich, M. T. The Missing Link: The Role of Interhemispheric Interaction in Attentional Processing. Brain Cogn. 36, 128-157 (1998).

52. Niogi, S., Mukherjee, P., Ghajar, J. \& McCandliss, B. D. Individual Differences in Distinct Components of Attention are Linked to Anatomical Variations in Distinct White Matter Tracts. Front. Neuroanat. 4, (2010).

53. Chechlacz, M., Humphreys, G. W., Sotiropoulos, S. N., Kennard, C. \& Cazzoli, D. Structural Organization of the Corpus Callosum Predicts Attentional Shifts after Continuous Theta Burst Stimulation. J. Neurosci. 35, 15353-15368 (2015).

54. Hornak, J. et al. Reward-related reversal learning after surgical excisions in orbito-frontal or dorsolateral prefrontal cortex in humans. J. Cogn. Neurosci. 16, 463-478 (2004).

55. Chudasama, Y. \& Robbins, T. W. Dissociable contributions of the orbitofrontal and infralimbic cortex to pavlovian autoshaping and discrimination reversal learning: further evidence for the functional heterogeneity of the rodent frontal cortex. $J$. Neurosci. Off. $J$. Soc. Neurosci. 23, 8771-8780 (2003).

56. Schoenbaum, G., Nugent, S. L., Saddoris, M. P. \& Setlow, B. Orbitofrontal lesions in rats impair reversal but not acquisition of go, no-go odor discriminations. Neuroreport 13, $885-890$ (2002). 
57. Ozalay, O. et al. The relationship between the anterior corpus callosum size and prefrontal cortex volume in drug-free depressed patients. J. Affect. Disord. 146, 281-285 (2013).

58. Putnam, M. C., Wig, G. S., Grafton, S. T., Kelley, W. M. \& Gazzaniga, M. S. Structural Organization of the Corpus Callosum Predicts the Extent and Impact of Cortical Activity in the Nondominant Hemisphere. J. Neurosci. 28, 2912-2918 (2008).

59. Tsang, H. T. H. et al. The hereditary spastic paraplegia proteins NIPA1, spastin and spartin are inhibitors of mammalian BMP signalling. Hum. Mol. Genet. 18, 3805-3821 (2009).

60. Wang, X., Shaw, W. R., Tsang, H. T. H., Reid, E. \& O'Kane, C. J. Drosophila spichthyin inhibits BMP signaling and regulates synaptic growth and axonal microtubules. Nat. Neurosci. 10, 177-185 (2007).

61. Xie, H. et al. Functional study of NIPA2 mutations identified from the patients with childhood absence epilepsy. PloS One 9, e109749 (2014).

62. Xiong, Y. \& Oakley, B. R. In vivo analysis of the functions of $\gamma$-tubulin-complex proteins. J. Cell Sci. 122, 4218-4227 (2009).

63. Laviola, G., Hannan, A. J., Macrì, S., Solinas, M. \& Jaber, M. Effects of enriched environment on animal models of neurodegenerative diseases and psychiatric disorders. Neurobiol. Dis. 31, 159-168 (2008).

64. Hoyer, C., Gass, N., Weber-Fahr, W. \& Sartorius, A. Advantages and Challenges of Small Animal Magnetic Resonance Imaging as a Translational Tool. Neuropsychobiology 69, 187-201 (2014).

65. Shi, Y. \& Toga, A. W. Connectome imaging for mapping human brain pathways. Mol. Psychiatry 22, 1230-1240 (2017). 
66. Leemans, A., Jeurissen, B., Sijbers, J. \& Jones, D. K. ExploreDTI: a graphical toolbox for processing, analyzing, and visualizing diffusion MR data. in 17th Annual Meeting of Intl Soc Mag Reson Med 209, 3537 (2009).

67. Calabrese, E., Badea, A., Watson, C. \& Johnson, G. A. A quantitative magnetic resonance histology atlas of postnatal rat brain development with regional estimates of growth and variability. NeuroImage 71, 196-206 (2013). 


\section{Methods}

Rats. The Cyfipl rat model was created by Cardiff University in collaboration with Horizon Discovery (St Louis, USA) using CRISPR-Cas9 targeting

(https://www.horizondiscovery.com/) and supported by a Wellcome Trust Strategic Award (DEFINE). Full information on the creation and validation of the rat model is in the Supplementary Methods section. All the rats used in this study were Long Evans males. The rats were produced from breeding stocks held at Charles River (UK) using a WT x HET design resulting in an average 1:1 WT to $C y f i p 1^{+/}$, the mutation was transmitted in Mendelian fashion with no sex bias and the rats were healthy and viable showing no general ill effects of the mutation. The rats were transported to Cardiff at 8-10 weeks of age. At Cardiff the rats were housed in mixed-genotype groups of 2-3 rats. The rats had free access to food and water (except for those used in the reversal learning task, see below) and lived under the condition of a $12 \mathrm{hr}$ light/day cycle (lights on at 7:00 am), room temperature $21 \pm 2$ ${ }^{\circ} \mathrm{C}$. Rats used in DTI were 5 months old. The rats were euthanized 1 month after the scanning and used for immunofluorescence. The rats used for electron microscopy were 6 months old. The rats used for behavioural experiments were 6-9 months old. The reversal learning task was motivated by liquid reward ( $10 \%$ sucrose solution $\mathrm{w} / \mathrm{v})$ and to enhance working in the task, the rats were subject to water restriction immediately prior to and during task training, in which case the rats were given 2 hours access to water per day. The water restriction schedule has no adverse effects on the health or welfare of the rats, being designed to give rise to a temporary increase in motivation for the liquid reinforcement, and across the whole day the rats on the schedule drink as much fluid as under free access conditions. All the experimental procedures were performed in accordance with institutional animal welfare and ARRIVE guidelines and the UK Home Office License PPL 30/3135. 
Diffusion tensor imaging acquisition. A cohort of 24 rats (WT $n=12$ and $C y f i p 1^{+/-} n=12$ ) were anaesthetised with isoflurane in oxygen at $4 \%$, and maintained at $1 \%$ during the scanning. MRI scans were acquired with a 9.4T MRI scanner (Bruker, Karlsruhe, Germany) with a $30-\mathrm{cm}$ bore and a gradient strength of up to $600 \mathrm{mT} / \mathrm{m}$. The MRI protocol included DTI acquisition with a diffusion-weighted (DW) spin-echo echo-planar-imaging (EPI) pulse sequence having the following parameters: TR/TE $=4000 / 22 \mathrm{~ms}, \Delta / \delta=10.5 / 4.5 \mathrm{~ms}$, two EPI segments, and 60 noncollinear gradient directions with a single b-value shell at $1000 \mathrm{~s} / \mathrm{mm}^{2}$ and one image with a b-value of $0 \mathrm{~s} / \mathrm{mm}^{2}$ (referred to as b0). Geometrical parameters were: 34 slices, each $0.32 \mathrm{~mm}$ thick (brain volume) and with in-plane resolution of $0.32 \times 0.32 \mathrm{~mm}^{2}$ (matrix size $80 \times 96$; FOV $25.6 \times 30.73 \mathrm{~mm}^{2}$ ). The DTI protocol lasted approximately 16 minutes. In addition, high resolution, T2 weighted images were acquired for anatomical reference with a multi-slice multi-echo pulse sequence with the following parameters: TR of $7200 \mathrm{~ms}$, TE of $15 \mathrm{~ms}$ and effective TE of $45 \mathrm{~ms}$, rare factor was 8 . Image resolution was set to $0.22 \mathrm{~mm}^{3}$ with matrix size of $128 \times 160 \times 50$ to cover the entire brain.

DTI data correction and DTI maps extraction. ExploreDTI 4.8.3 ${ }^{66}$ and SPM (version 12, UCL, London, UK) were used in the preprocessing of the rat DTI data. First, eddy-current induced distortion and motion correction were performed, and mean-DWI images were extracted using ExploreDTI. Non-brain tissue was removed from the mean-DWI and the $\mathrm{T}_{2}$ weighted images following these steps: (1) $T_{2}$-weighted scans were anisotropic smoothed using ExploreDTI, (2) both smoothed $\mathrm{T}_{2}$-weighted and mean DWI images were bias corrected using the segmentation tool in SPM12, (3) the bias corrected $\mathrm{T}_{2}$-weighted were coregistered with a population-specific template and multiplied by a mask to remove the non- 
brain tissue, (4) the skull was removed from the mean DWIs using the 3D masking option in ExploreDTI. Then, data was corrected for field inhomogeneities, using ExploreDTI, where the skull-stripped mean DWIs images were used as a native space mask, and the skullstripped $\mathrm{T}_{2}$-weighted structural scans were used as transformed space mask. Each DWI image was nonlinearly warped to the $\mathrm{T}_{2}$-weighted image using non-DWIs map as a reference. ExploreDTI was used to generate FA, AD, RD and MD maps.

Preprocessing for Tract-Based Spatial Statistics (TBSS). For the voxel-wise analyses of DTI data, Tract-Based Spatial Statistics (TBSS) method was implemented, which is part of the FMRIB software library (FSL). All FA maps were submitted to a free-search for a best registration target, where each volume was first registered to every other volume, and the one requiring minimum transformation to be registered to other volumes was selected as the best registration target. This target was used as a template into which the registration was performed. Following registration, a mean FA map was calculated, thinned to represent a mean FA skeleton, and an optimal threshold of 0.2 was applied to the mean FA skeleton to create a binary white matter skeleton mask (Supplementary Figure 1). The local FA-maxima, as well as the $\mathrm{AD}, \mathrm{RD}$, and $\mathrm{MD}$, of each rat were projected onto this white matter skeleton.

Transmission electron microscopy and immunofluorescence. For transmission electron microscopy, a new cohort of 9 rats (WT n=5 and $C y f i p 1^{+/-} \mathrm{n}=4$ ) was used. For immunofluorescence seven brains were randomly selected (WT $n=7$ and $C y f i p 1^{+/-} n=7$ ) from the cohort used for DTI. In both cohorts, the rats were intracardially perfused with $0.1 \mathrm{M}$ phosphate buffered saline (PBS), followed by $4 \%$ of glutatradehyde in $0.1 \mathrm{M}$ PBS in the cohort used for electron microscopy, and 4\% paraformaldehyde in $0.1 \mathrm{M}$ PBS (PFA) in the cohort used for immunofluorescence. For transmission electron microscopy, the brains 
were placed on a shaker to postfix in glutatradehyde for $4 \mathrm{~h}$, after which they were placed in phosphate buffered saline and stored at $4 \mathrm{C}$ until further use. Then the brains were embedded in $\mathrm{TAAB}$ embedding resin. Ultra-thin sections $(50 \mathrm{~nm})$ were stained with aqueous $4 \%$ uranyl acetate and lead citrate. The sections were visualized on a transmission electron microscope (CM12, Philips, the Netherlands) and, for quantification, images were taken using an on-axis $2048 \times 2048$ charge-coupled device camera (Proscan, Schering, Germany). In order to obtain a representative sample, 15 regions across the extent of the anterior-posterior extent of the corpus callosum per animal were taken for quantification. For immunofluorescence, the brains were placed on a shaker to postfix in PFA for $4 \mathrm{~h}$, after which they were placed in phosphate buffered $30 \%$ sucrose. Coronal cryosections of the brain, of $15 \mu \mathrm{m}$ thickness, were made on a cryostat (CM1860 UV, Leica, UK), mounted onto a polylysine coated slides (3 sections per slide), and stored at $-20^{\circ} \mathrm{C}$. For immunofluorescence, antibodies were used as follows: anti-Olig2 (ab109186, Abcam) 1:400, anti-APC [CC-1] (ab16794, Abcam) 1:400, anti-MBP (MAB386, Millipore) 1:300. For the Olig2 and Cc1 doublestaining, the slices were heated in a 5\% citrate buffered antigen retrieval solution $(\mathrm{pH} 6,10 \mathrm{x}$, Sigma-Aldrich Company, UK), using a water bath at $90 \%$ for $10 \mathrm{~min}$. All the slices were blocked for 1 hour with 5\% donkey serum (Sigma-Aldrich Company, UK), and 0.3\% Triton X-100 in PBS. The appropriate primary antibodies were applied and incubated overnight at $4{ }^{\circ} \mathrm{C}$. On the next day, after washing, the slices were incubated for 2 hours with secondary antibodies (Alexa Fluor Life Technologies, Manchester, UK), in a concentration of 1:1000 at room temperature. Then, the slides were washed, counterstained with 1:1000 DAPI, mounted and cover-slipped. For quantification of Olig2 + and $\mathrm{Cc} 1+$ cells, images were taken on an inverted fluorescent time lapse microscope (DMI6000B, Leica, UK), and at least 4 images from random visual fields were taken from regions including the corpus callosum and external capsule. For 
quantification of MBP intensity, one coronal section per rat was taken on an Axio scan (Zeiss, Germany), and the same exposure time and intensity were used for all the slides.

Reversal Learning. A separate group of 17 rats were used in the reversal learning task (WT $\mathrm{n}=7$, Cyfip $1^{+/-} \mathrm{n}=10$ ). Testing was conducted in a touchscreen-based automated operant system that consisted of an operant chamber with a flat-screen monitor equipped with an infrared touchscreen with accompanying Animal Behaviour Environment Test (ABET) II software (Campden Instruments, Leics). Session duration was 30 minute, or until 100 trials were completed under all training conditions. Pre-training consisted of two stages (Magazine Training and Touch Training) these gradually shaped the screen-touching behaviour required for the reversal learning touchscreen task proper. Following successful completion of pretraining Visual Discrimination Training began (Supplementary Figure 4); two stimuli were presented at a time (S+ and S-, counterbalanced across animals), on either side of the screen. The rat had to touch the correct stimulus $(\mathrm{S}+)$ to elicit reward. Reward delivery was accompanied by illumination of the tray light and a tone. Entry to collect the reward turned off the tray light and started the inter-trial interval (ITI $-5 \mathrm{~s}$ ) following which the rat initiated the next trial by a second magazine entry. Touching the incorrect stimulus (S-) terminated the trial and the house-light was turned on for a time-out period of $5 \mathrm{~s}$ and no reward given, following the time-out the ITI period began after which the rat had to initiate the next trial by executing a magazine entry. Once the rats reached performance criteria (Completing 50+ trials with $80-85 \%$ correct, for 2 consecutive sessions), the contingencies were reversed (previous S+ now S-; previous S- now S+) and behaviour monitored (see Supplementary Figure 4 for schematic of task design). 
Mismatch Task. A different group of 36 rats performed the mismatch task (WT n=21, Cyfip $\left.1^{+/-} \mathrm{n}=15\right)$. On the first 4 days, the rats were placed in the experimental apparatus (a modified skinner box allowing presentations of auditory and visual stimuli) for $30 \mathrm{~min}$. Following this general habituation to the apparatus they received 4 days of training with two audiovisual sequences. One auditory stimulus (a $2 \mathrm{kHz}$ tone) preceded the constant presentation of a light, whereas a second auditory stimulus (a $10 \mathrm{~Hz}$ series of clicks) preceded the flashing presentation of the same light stimuli (i.e. Tone $\rightarrow$ Steady Light, Click $\rightarrow$ Flashing Light; the combinations were randomly counterbalanced across animals). All stimuli were presented for $10 \mathrm{sec}$. There were 10 presentations of both audiovisual sequences on each of the first 3 days of training and six presentations of both sequences on day 4 that served as warm-up trials for the eight test trials that immediately followed. The inter-trial-interval was $2 \mathrm{~min}$. Rats received two types of test trials, match and mismatch. The order in which the two types of test trials were presented was counterbalanced. Match test trials were presentations of the same audiovisual sequences that had been presented during training (e.g.

Tone $\rightarrow$ Steady Light, Click $\rightarrow$ Flashing Light), whereas on mismatch trials the auditory stimuli preceding the visual stimuli were exchanged (e.g. Tone $\rightarrow$ Flashing Light, Click $\rightarrow$ Steady Light). All experimental sessions were recorded using a video recorder and orienting responses subsequently scored by observers who were blind to the genotype of the rats and the nature of the test trials (match or mismatch). An Orienting Response (OR) was defined as the tip of a rat's snout being located in the side of the apparatus that contained the light and pointing in the direction of the light.

Statistical analyses. Differences in DTI measures between the two groups (WT and Cyfip1 $1^{+-}$ ) were assessed using voxel-wise independent t-tests, where two different contrasts were used $\left(\mathrm{WT}>C y f i p 1^{+/-}\right.$, and $\left.C y f i p 1^{+/-}>\mathrm{WT}\right)$. Using the randomize function (part of FSL), the null 
distribution was built over 1000 random permutations, using the Threshold-Free Cluster Enhancement (TFCE) ${ }^{25}$ algorithm where cluster-like structures are enhanced, and the results are shown for $\mathrm{p}<0.05$. For multiple comparison correction, first FWE correction was used. Since only FA changes were found within this analysis, we also used a less conservative correction method based on FDR correction, purposed by Benjamini-Hochberg ${ }^{26}$. To quantify the changes in areas where significant differences in FA were seen after FWE correction, regions of interest (ROIs) were manually delineated using FSL. Several consecutive slices were outlined on the coronal plane and the selected ROIs included the corpus callosum, internal capsule, external capsule, and fornix/fimbria regions. The CBJ13 MR-histology rat atlas at age $\mathrm{P} 80^{68}$ was used as reference. A representation of the binary masks can be found in Supplementary Figure 2. FA, AD, RD, and MD were quantified by applying these binary masks and extracting the mean values for each region across subjects.

For quantification of cells the ImageJ software (version 1.51) was used. The number of myelinated and unmyelinated axons, axon diameter, myelin thickness and g-ratio (measure of myelin thickness relative to axon diameter: where lower g-ratios indicates thicker myelin sheath) of normally myelinated axons were quantified. A total of 13128 (WT $n=7148$,

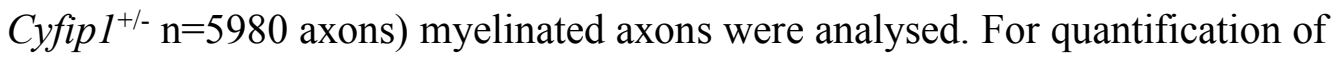
oligodendrocytes, the total number of Olig2 + , and the overlapped Olig2 $+/ \mathrm{Cc} 1+$ cells were counted. Only cell bodies clearly identified by Olig2 and Cc1 immunofluorescence and overlapping with DAPI staining were counted. The number of cells were divided by the area quantified in each image. $\mathrm{MBP}+$ reactivity was determined by comparing immunofluorescence staining intensity. The whole region of corpus callosum and external capsule was selected in the coronal section, and quantification was done by calculating the mean intensity of the pixels above a preset intensity threshold, multiplied by the number of 
pixels above that threshold, and dived by the total area quantified. All the analyses were conducted with the investigator blinded to the phenotype. Differences between WT and Cyfip $^{+/-}$were analysed in RStudio. In order to compare all the axons in each group while taking into account variation across individuals, we used linear mixed effects models to analyse the effect of genotype on axon diameter, g-ratio and myelin thickness, where these measures were considered fixed effects, and animals were considered random effects. Since we only had one random effect, we used non-restricted maximum likelihood to estimate the model parameters. In this analysis, the myelin thickness was log-transformed since the data followed a log-normal distribution, whereas the other measures followed a normal distribution. All the other measures were analysed using two-tailed unpaired Student's t-test. Data are given as mean \pm s.e.m.

Visual discrimination and reversal learning performance was assessed using ANOVA with factors of GENOTYPE and SESSION. Any significant interaction was subsequently examined by analysing the Simple Effects. Completion rates for the rats during the different phases of reversal were assessed non-parametrically using Chi-squared test. Performance in the mismatch task was assessed using ANOVA with factor of GENOTYPE and BLOCK during the habituation to the test apparatus phase of training and factor GENOTYPE in the habituation to the stimulus pairs and mismatch test phases. Orienting responses in the mismatch test phase were analysed as a discrimination ratio (total orienting to matched / total orienting to both matched + mismatched). 